\title{
HE4 (WFDC2) gene overexpression promotes ovarian tumor growth
}

SUBJECT AREAS:

CELL BIOLOGY

DRUG DISCOVERY

MOLECULAR BIOLOGY

CANCER

Received

17 June 2013

Accepted

5 December 2013

Published

6 January 2014

Correspondence and requests for materials should be addressed to

R.G.M. (rmoore@ wihri.org) or R.K.S. (rsingh@wihri.org)
Richard G. Moore', Emily K. Hill', Timothy Horan', Naohiro Yano², KyuKwang Kim', Shannon MacLaughlan ${ }^{3}$, Geralyn Lambert-Messerlian ${ }^{4}$, YiTang Don Tseng ${ }^{2}$, James F. Padbury², M. Craig Miller' ${ }^{1}$, Thilo S. Lange ${ }^{1,5}$ \& Rakesh K. Singh ${ }^{1}$

'Molecular Therapeutics Laboratory, Program in Women's Oncology, Women and Infants' Hospital of Rhode Island, Alpert Medical School, Brown University, Providence, RI 02903, USA, ²Department of Pediatrics, Women and Infants' Hospital of Rhode Island, Alpert Medical School, Brown University, Providence, RI 02903, USA, ${ }^{3}$ Stanford School of Medicine, Gynecology Oncology, 300 Pasteur Dr. A370 Stanford, CA 94305, USA, ${ }^{4}$ Prenatal and Special Testing, Department of Pathology, Women and Infants' Hospital of Rhode Island, Alpert Medical School, Brown University, Providence, Rhode Island, USA, ${ }^{5}$ Department of Molecular Biology, Cell Biology, and Biochemistry, Brown University, Providence, RI 02912, USA.

Selective overexpression of Human epididymal secretory protein E4 (HE4) points to a role in ovarian cancer tumorigenesis but little is known about the role the HE4 gene or the gene product plays. Here we show that elevated HE4 serum levels correlate with chemoresistance and decreased survival rates in EOC patients. HE4 overexpression promoted xenograft tumor growth and chemoresistance against cisplatin in an animal model resulting in reduced survival rates. HE4 displayed responses to tumor microenvironment constituents and presented increased expression as well as nuclear translocation upon EGF, VEGF and Insulin treatment and nucleolar localization with Insulin treatment. HE4 interacts with EGFR, IGF1R, and transcription factor HIF $1 \alpha$. Constructs of antisense phosphorothio-oligonucleotides targeting HE4 arrested tumor growth in nude mice. Collectively these findings implicate increased HE4 expression as a molecular factor in ovarian cancer tumorigenesis. Selective targeting directed towards the HE4 protein demonstrates therapeutic benefits for the treatment of ovarian cancer.
$\mathrm{H}$ uman epididymis protein 4 (HE4), also called whey-acidic-protein (WAP) four-disulfide core domain protein 2 (WFDC2) was initially described to have tissue specific expression in the epididymis ${ }^{1}$. Clinical research in the last decade revealed that HE4 is expressed in a limited number of other organs, including the female reproductive tract, breast tissue, kidney, regions of the respiratory tract and nasopharynx ${ }^{2-4}$. HE4 in human ovarian cancer cells is produced as a $\sim 13 \mathrm{kD}$ protein and converted to a $\sim 25 \mathrm{kD}$ secreted glycosylated protein. HE4 (WFDC2) is highly overexpressed in epithelial ovarian cancer (EOC) ${ }^{5-8}$ compared to normal ovarian epithelium and the measurement of serum HE4 levels in women with EOC has been shown clinical relevance. The USFDA cleared HE4 as a biomarker for the detection of ovarian cancer in women presenting with an ovarian cyst or pelvic mass as part of the Risk of Ovarian Malignancy Algorithm (ROMA) and for monitoring women diagnosed with $\mathrm{EOC}^{9-13}$. Overexpression of Human epididymal secretory protein E4 (HE4) in EOC points to a role in ovarian cancer tumorigenesis, however little is known about the biological functions of the HE4 gene or its gene product. Here we show that elevated HE4 serum levels correlate with chemoresistance and decreased survival rates in EOC patients and demonstrate that HE4 overexpression promotes ovarian tumor growth in an animal model. We also demonstrate that HE4 interacts with growth factors and oncogenes previously linked to ovarian tumor growth and chemoresistance. Finally, we show that antisense inhibition of HE4 via novel phosphorothio-oligonucleotides (PTOs) resulted in reduced ovarian cancer cell viability and suppressed growth of xenografted tumors in mice. Taken together, our studies provide evidence that HE4 overexpression plays an important role in ovarian tumor growth and chemoresistance.

\section{Results}

HE4 expression levels correlate with lower survival and chemoresistance in human ovarian cancer patients. To delineate the correlation of serum HE4 levels and chemoresistance in women with EOC we investigated the association of pre-operative serum HE4 levels with chemosensitivity and survival in a retrospective study of 89 women with EOC at Women and Infants Hospital (Institutional Review Board approval:11-005). Patients were stratified based on preoperative serum HE4 levels. Survival curves were plotted and Cox hazards regression 
analysis was used to determine association between prognostic variables and overall survival (OS). The median OS at 5 years was $53.9 \%$. Women with a serum HE4 level $\geq 500 \mathrm{pM}$ had a 5 -year OS of $27 \%$ compared to $59 \%$ for those with HE4 $<500$ pM $(\mathrm{p}=0.005)$ (Fig. 1A). Median OS analysis for high HE4 versus low HE4 expressers revealed a Hazard Ratio (HR) of 2.2 (95\% CI: 1.3 - 3.9; $\mathrm{p}=0.005)$. Examination of the Risk of Ovarian Malignancy Algorithms (ROMA) scores, which employs serum levels of HE4 and CA125 along with menopausal status to predict the presence of ovarian cancer, showed that women with a ROMA score $\geq 60 \%$ had a 5-year survival rate of $38 \%$ and those with a ROMA score $<60 \%$ had a $76 \% 5$-year survival rate $(\mathrm{p}=0.003)$. Likewise analysis of median OS provided a HR of 3.3 (95\%CI: $1.4-7.9 \%$; $p=0.0014$ ) (Fig. 1B). In correlation with these findings, patients with platinum resistant disease had a 5 years survival rate of $29 \%$ compared with $57 \%$ for patients with platinum sensitive disease $(\mathrm{p}=0.015)$. Analysis of median OS in the platinum resistant group versus the platinum sensitive group provided a HR of 2.0 (95\%CI: $1.3-3.8 ; \mathrm{p}=0.0068$ ). Within two years, the platinum resistant group witnessed a threefold higher death rate of $52 \%$ compared with the platinum sensitive group with a rate of $14 \%(p=0.001)$ (Fig. 1C). In concordance with our observations of the correlation between HE4 over expression and

A

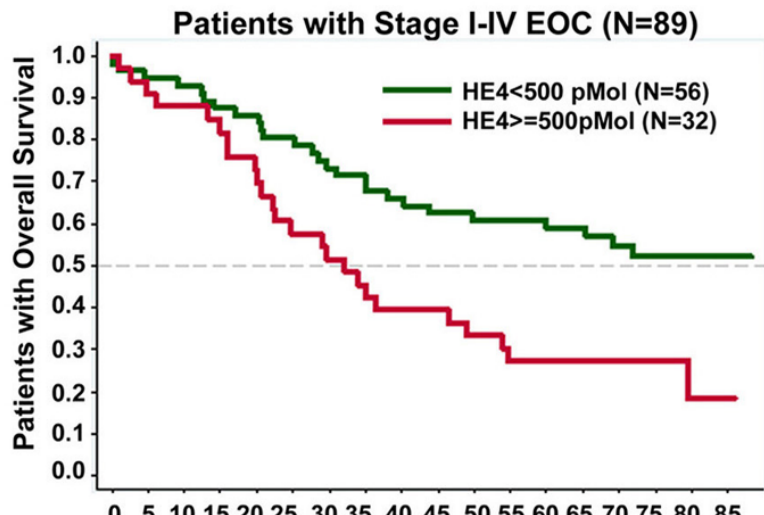
Number at risk

Time from Pre-Operative Blood Draw [months] HE4=<500 $5653524948454140 \quad 373534343431241713 \quad 6$

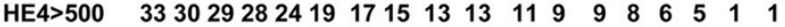

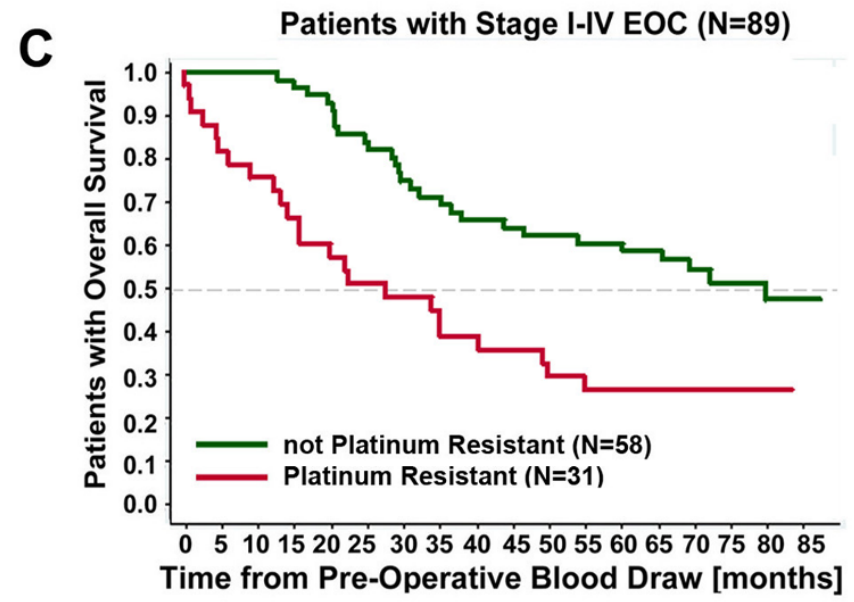

Number at risk

$\begin{array}{lllllllllllllllllll}\text { CA125<100 } & 58 & 58 & 58 & 57 & 54 & 49 & 43 & 41 & 38 & 37 & 35 & 34 & 34 & 31 & 22 & 16 & 11 & 6\end{array}$ $\begin{array}{lllllllllllllllllll}\mathrm{CA} 125>=100 & 31 & 25 & 23 & 20 & 18 & 15 & 15 & 14 & 12 & 11 & 10 & 9 & 9 & 8 & 8 & 6 & 3 & 0\end{array}$ poor patient survival rates, CA125 overexpression with serum levels $\geq 100 \mathrm{U} / \mathrm{ml}$ was also associated with lower 5 year survival rates compared with patients that had serum levels $<100 \mathrm{U} / \mathrm{ml}$ (58 vs $41 \%$ ). Median OS analysis provided a HR of HR:1.9 (95\%CI: 1.0-3.6; $\mathrm{p}=0.046$ ) (Fig. 1D). However, HE4 and ROMA scores emerged as more sensitive predictors of survival and chemoresistance than CA125 alone. Similarly, stage (stage I \& II versus stage III \& IV) [HR 4.4 (95\% CI: 1.7-11.0), $\mathrm{p}=0.0002$ ], and postmenopausal vs menopausal [HR: 3.0, (95\% CI: 0.9-9.6), $p=0.0297$ ] was also associated with higher risk of death and poor survival (Supplementary Fig. 1).

Stable HE4 overexpression promotes cisplatin resistance in ovarian cancer cells and tumor growth in nude mice. To study the biological role of HE4 overexpression in ovarian cancer tumorigenesis and chemoresistance, stable HE4 overexpressing SKOV-3 (HE4C1, HE4C7) and OVCAR-8 (HE4C5) ovarian cancer cell clones were developed (see methods). OVCAR-8 and SKOV-3 cells are both derived from ovarian epithelial adenocarcinoma and are resistant to platinum based chemotherapeutics which are the standard of care for ovarian cancer treatment. HE4 levels were measured in cell lysates and media (Fig. 2A). Evaluation of the cell

B

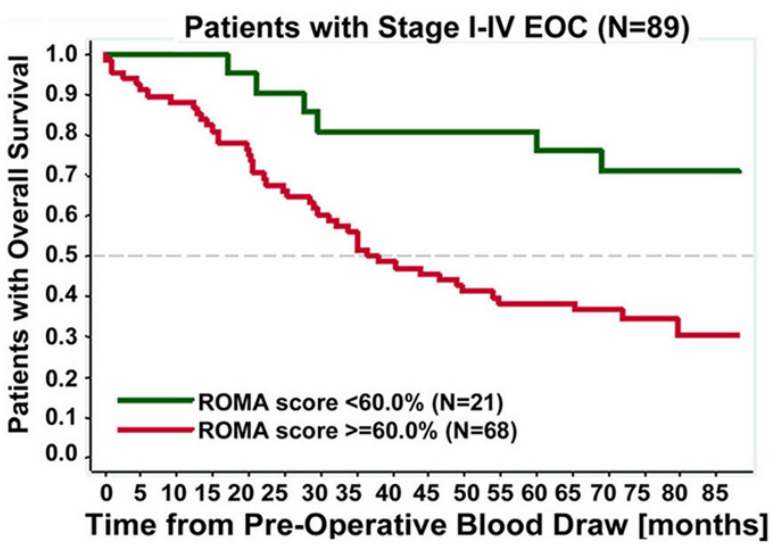

Number at risk

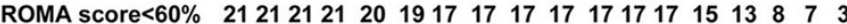
ROMA score $>=60 \% \quad 68 \quad 62605652 \quad 4541 \quad 38 \quad 33 \quad 3128 \quad 2626 \quad 24 \quad 17 \quad 14 \quad 7 \quad 3$

D

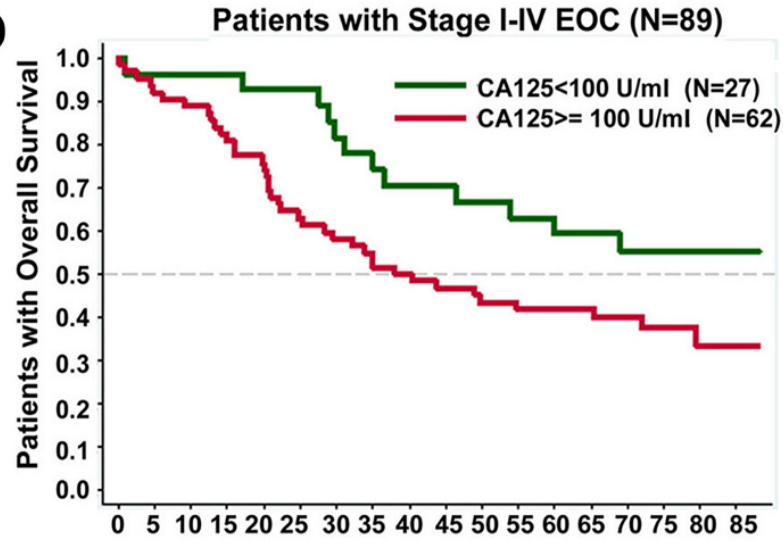

Time from Pre-Operative Blood Draw [months]

Number at risk

CA125<100 $272626262525222119191817171613 \quad 8 \quad 7 \quad 4$

CA125>=100 $62 \quad 5755 \begin{array}{llllllllllllllll}40 & 39 & 36 & 34 & 31 & 29 & 27 & 26 & 26 & 23 & 17 & 14 & 7 & 2\end{array}$

Figure 1 Survival analysis of ovarian cancer patient cohort. (A) Kaplan-Meier survival curve of 89 ovarian cancer patients (all stages) show decreased survival for patients with HE4 $>500$ pM compared with patients with HE4 $<500$ pM. (B) Patients with ROMA scores $>60 \%$ have decreased survival compared with patients that have ROMA scores $<60 \%$. (C) Clinical assessment showing Platinum resistant tumors affect patient survival. (D) Patients with CA125>100 U/ml experience higher mortality than those with CA125 <100 U/ml. 
A
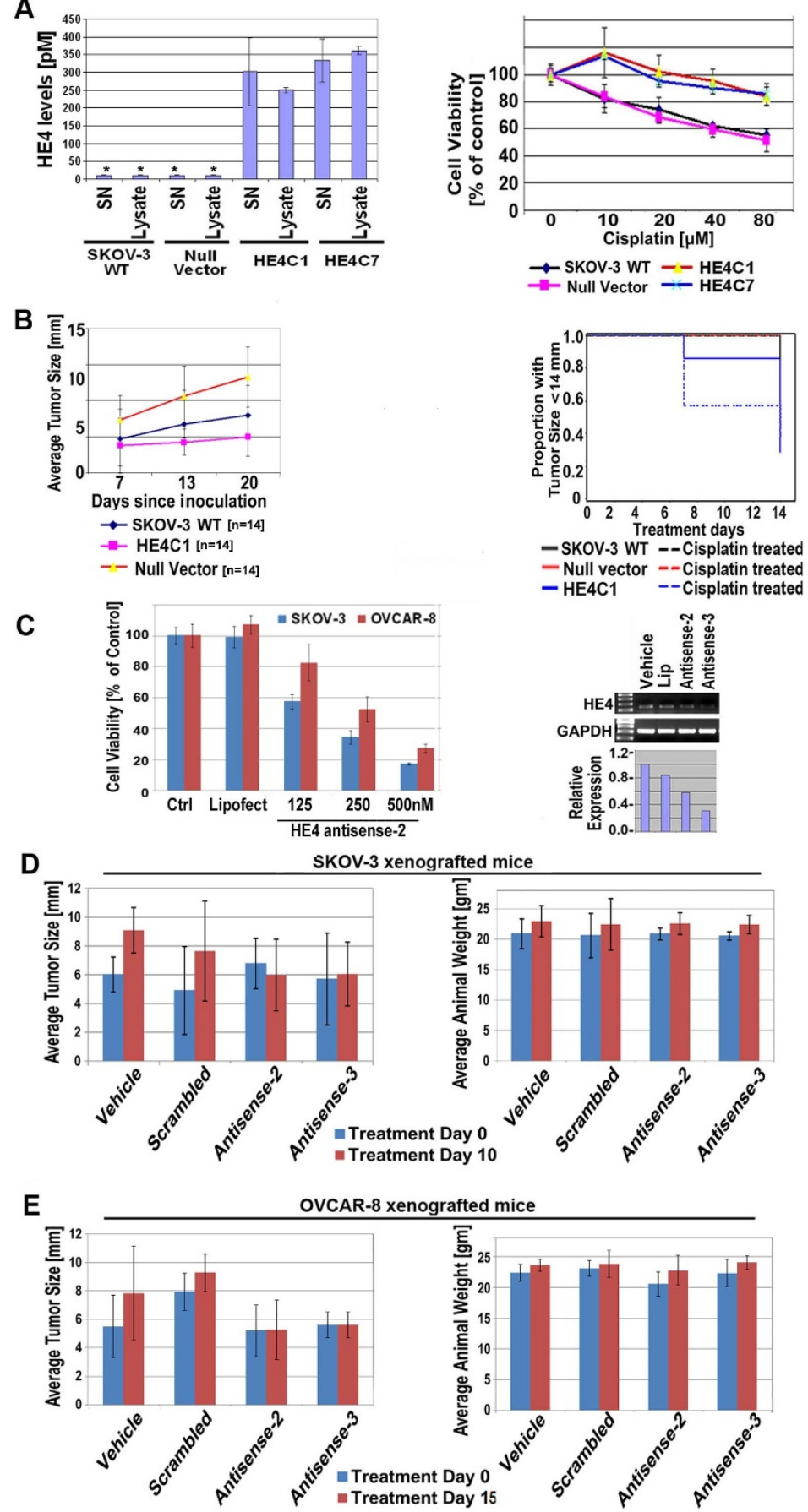

Figure $2 \mid$ HE4 overexpression promotes tumor growth and chemoresistance against cisplatin. (A) HE4 cellular (lysate) and media secretion (SN; supernatant, $24 \mathrm{~h}$ ) levels in wild type SKOV-3, null vector clones and stably HE4 overexpression SKOV-3 clones (HE4C1, HE4C7) were determined by ELISA (left panel), * indicates HE4 values below 15pM. MTS based cell viability assay of WT SKOV-3, null vector and HE4 overexpressing clones treated with Cisplatin for $24 \mathrm{~h}$ revealed increased resistance by HE4 overexpressing cells. (right panel). (B) Tumor sizes of WT SKOV-3, null vector and SKOV-3 $\mathrm{HE} 4 \mathrm{C} 1$ clone derived xenografts differed significantly during 20 days of trial (left panel). A Tukey-adjusted pairwise group difference showed that tumor size differed between SKOV-3 HE4C1 vs WT $(\mathrm{p}=0.007)$ and HE4C1 vs empty vector $(\mathrm{p}<0.0001)(\mathrm{n}=14$ each). There was no significant tumor size difference between the WT and null vector clone $(\mathrm{p}=0.3$ ). Upon treatment with cisplatin for 14 days (days 20-34 from inoculation) cisplatin resistance and aggressive growth of HE4 overexpressing tumors was observed. Kaplan-Meier curve showed that HE4C1 derived tumors treated with cisplatin experienced excessive growth compared to cisplatin/vehicle treated WT SKOV-3 and null vector or HE4C1 treated with vehicle. Group difference among cisplatin treated groups was significant $(\mathrm{p}=0.007)$ and vehicle treated group showed $\mathrm{p}=0.007$ (right panel). (C) MTS viability assay of SKOV-3 and OVCAR-8 cell-lines treated with novel HE4 antisense phosphoro-thio-oligonucleotides (PTO) for 24 hours (left panel). RT-PCR of SKOV-3 mRNA showed that Antisense-2 and Antisense-3 PTO reduced expression of HE4 (right panel) (D, E) Antisense-2 and Antisense-3 treatment ( $7 \mathrm{mg} / \mathrm{kg}$, IP daily) stopped SKOV-3 (D, left panel; 10 day treatment) and OVCAR-8 (E, left panel; 15 day treatment) xenograft tumor growth in nude nice. PTO treatment) did not affect weight of animals xenografted with SKOV-3 (D, right panel) and OVCAR-8 (E, right panel). 
lysate and Media showed that SKOV-3 HE4C1 produced average $302.6 \mathrm{pM}$ and secreted 250.6pM of HE4. Similarly, HE4C7 produced average $333.6 \mathrm{pM}$ and secreted $362.3 \mathrm{pM}$ of HE4 (Fig. 2A, left panel) and displayed increased HE4 mRNA expression (Supplementary Fig. 2A) compared with Null vector transformed and wild type SKOV-3 which had non-detectable HE4 levels $(<15 \mathrm{pM})$. A MTS based in vitro cell viability assay demonstrated that $\mathrm{HE} 4 \mathrm{C} 1$ and HE4C7 tumor cell lines were less sensitive to cisplatin (Fig. 2A, right panel) and paclitaxel treatment (Supplementary Fig. 2B) in vitro compared with controls. Similarly, HE4 overexpressing OVCAR-8 HE4C5 clones (Supplementary Fig. 2C) showed increased resistance to cisplatin (Supplementary Fig. 2D).

Next, we determined the effect of HE4 overexpression in ovarian cancer tumor growth in vivo. Mice xenografted with SKOV-3 HE4C1 showed aggressive tumor growth compared to the null vector and wild type SKOV-3 xenografted tumors (Fig. 2B, left panel). On day 20 the average tumor size in the $\mathrm{HE} 4 \mathrm{C} 1$ group was $11 \mathrm{~mm}$ compared with $5-6 \mathrm{~mm}$ in the null vector and wild type SKOV-3 group. Similarly, Li et al. recently reported that HE4 overexpression promotes tumor growth in endometrial cancer cell lines and a xenograft mouse model ${ }^{14}$. Tukey-adjusted pairwise group difference analysis examining the tumor size between $\mathrm{HE} 4 \mathrm{C} 1$, null vector and wild type derived tumors showed significantly larger tumor size for HE4C1 versus WT ( $p=0.007$ ) as compared to the control groups versus null vector $(\mathrm{p}<0.0001)$. There was no significant difference in tumor size between the wild type and null vector clones $(\mathrm{p}=0.3)$. To determine a potential role of HE4 in the response to standard therapeutics, animals in each group were subdivided on day 20 after inoculation with tumor cells and treated with vehicle or cisplatin $(10 \mathrm{mg} / \mathrm{kg})$ for 14 days (Fig. 2B, middle and right panel). All seven wild type SKOV-3 xenografted animals $(100 \%$; $7 / 7)$ treated with vehicle and $86 \%$ of animals (6/7) that received cisplatin displayed tumors smaller than $14 \mathrm{~mm}$ on day 34. In the SKOV-3 null vector group tumors size stayed below $14 \mathrm{~mm}$ for all animals $(100 \% ; 7 / 7)$ when treated with vehicle or cisplatin. In contrast, examination of the HE4 overexpressing $\mathrm{HE} 4 \mathrm{C} 1$ xenograft revealed that 5 of $7(78 \%)$ animals treated with cisplatin had tumors that grew larger than $14 \mathrm{~mm}$. Animals treated with vehicle in this group, only 2 of 7 (28\%) had tumors greater than $14 \mathrm{~mm}$. This indicates not only development of cisplatin resistance but enhanced cisplatin-induced tumor growth ${ }^{15}$ of HE4 overexpressing cells as visualized by Kaplan-Meier analysis (Fig. 2B, right panel). There was a significant difference observed in tumor growth among cisplatin or vehicle treated SKOV-3 HE4C1 groups $(\mathrm{p}=0.007)$.

Antisense targeting of HE4 suppressed ovarian cancer cell and tumor growth. To further investigate HE4 as a therapeutic target for the treatment of ovarian cancer, HE4-antisense phosphorothiooligonucleotides (PTO) were synthesized (IDT Inc.; Coralville, IA) [antisense-2: $5^{\prime} \mathrm{A}^{*} \mathrm{C}^{*} \mathrm{~A}^{*} \mathrm{C}^{*} \mathrm{C}^{*} \mathrm{~T}^{*} \mathrm{~T}^{*} \mathrm{C}^{*} \mathrm{C}^{*} \mathrm{C}^{*} \mathrm{~A}^{*} \mathrm{C}^{*} \mathrm{~A} * \mathrm{G}^{*} \mathrm{C}^{*} \mathrm{C}^{*} \mathrm{~A}^{*}$ T*T3'; antisense-3: $5^{\prime} G^{*} A^{*} C^{*} A^{*} C^{*} C^{*} T^{*}{ }^{*}{ }^{*}{ }^{*} C^{*} C^{*} A^{*} C^{*} A^{*} G^{*}$ $\left.\mathrm{C}^{*} \mathrm{C}^{*} \mathrm{~A}^{*} \mathrm{~T}^{*} \mathrm{~T} 3^{\prime}\right]$. With the knowledge that SKOV-3 and OVCAR-8 ovarian cancer cell lines display cisplatin resistance and express base line levels of HE4, cell viability and tumor growth was evaluated after treatment with HE4-antisense PTO. HE4 antisense PTO reduced the cell viability of SKOV-3 and OVCAR- 8 cells within 24 hours dose dependently (Fig. 2C, left panel). HE4 antisense PTO reduced the HE4 mRNA expression within $24 \mathrm{~h}$ (shown for SKOV-3; Fig. 2C, right panel). We evaluated the antitumor efficacy of antisense PTO in comparison to a scrambled PTO $\left(5^{\prime} \mathrm{C}^{*} \mathrm{~T}^{*} \mathrm{C}^{*} \mathrm{~A}^{*} \mathrm{G}^{*} \mathrm{G} * \mathrm{~A}^{*} \mathrm{~T} * \mathrm{G}^{*}\right.$ $\left.G^{*} C^{*} G^{*} G^{*} A^{*} G^{*} C^{*} G^{*} G^{*} T^{*} C^{*} T 3^{\prime}\right)$ in a subcutaneous xenograft model in nude mice. Within 10 days of daily $(7 \mathrm{mg} / \mathrm{kg}$, IP) administration, antisense- 2 and antisense-3 PTO arrested the growth of SKOV-3 xenograft tumors relative to scrambled PTO or vehicle (Fig. 2D, left panel). Similarly, 15 day treatment with antisense-2 and -3 PTO $(7 \mathrm{mg} / \mathrm{kg}$, IP) decreased tumor growth in OVCAR-8 xenografted animals (Fig. 2E, left panel). Treatment with antisense-2 and -3 did not cause any adverse effect on animal weight during studies (Fig. 2 D/E, right panels).

HE4 interaction with growth factor receptors and HIF1a. To delineate HE4 mediated signaling interactions, we examined SKOV-3 xenograft tissues histochemically, as these tissues provide a snapshot of a tumor microenvironment where soluble factors, proteins, and host-tumor immune mediators converge to support tumor growth. Confocal microscopy revealed cytoplasmic as well as nuclear staining of HE4 in SKOV-3 xenograft tissues (Fig. 3A, left panel). In contrast, cultured ovarian cancer cells (SKOV-3, OVCAR-8; Fig. 3A middle panels) demonstrated only cytosolic staining. We screened the effect of recombinant growth factors on SKOV-3 (EGF, VEGF, Insulin), and OVCAR-8 (EGF, Insulin) cells to determine changes in levels or the cellular localization of HE4 (treatment for $30 \mathrm{~min}$, see methods). The oncogenic role of these growth factors ${ }^{16,17}$ in ovarian tumorigenesis is well established. Confocal microscopy revealed nuclear translocation of HE4 upon treatment with the growth factors listed above (Fig. 3A, middle panels). In addition, increased cellular expression of HE4 upon stimulation with EGF, Insulin or VEGF was measured (Fig. 3A, right panel). Remarkably, stimulation with insulin displayed intense nucleolar localization of HE4 in OVCAR-8 cells (Fig. 3A, lower middle panel). The stimulation with VEGF did not cause nuclear accumulation of HE4 in OVCAR-8 cells.

EGF-stimulated HE4 nuclear translocation suggested a potential interaction of HE4 with EGFR or other cell surface receptors. A confocal microscopy of SKOV-3 xenograft tissue revealed strong co-localization of HE4 and EGFR (Pearson Coefficient $=\sim 0.9$ ) (Fig. 3B). Further, EGFR showed co-immunoprecipitation with HE4 (HE4 antibody as bait) as compared to IgG (Fig. 3C, left panel). Moreover, elevation in HE4 levels (e.g. in SKOV-3 HE4C1 and HE4C7 clones) elicited higher EGFR co-immunoprecipitation as compared to wild type SKOV-3 and a null vector clone. Measurement of intensity of HE4 expression after immuno staining was performed and revealed that treatment of cells with an EGF inhibitor (Iressa) inhibited EGF-induced HE4 overall staining in SKOV-3 cells. The measurements (relative intensity units/U) were as follows. No treatment: mean $615 \pm 130 \mathrm{U}$ (45 fields); EGF treated: mean $909 \pm 225 \mathrm{U}$ (40 fields); Iressa and EGF treated: mean 579 $\pm 113 \mathrm{U}$ (46 fields).

VEGF induced nuclear translocation of HE4 pointed to a potential correlation of HE4 expression with angiogenesis regulators such as HIF1 $\alpha$. SKOV-3 derived xenograft tissues showed strong co-localization of HIF1 $\alpha$ and HE4 (Fig. 3D). Nuclear expression of HIF1 $\alpha$ independently and in cohort with VEGF promotes aggressive and chemoresistant disease and denotes poor prognosis in ovarian cancer patients ${ }^{18-20}$. SKOV-3 xenograft tissue lysates showed co-immunoprecipitation of HIF1 $\alpha$ with HE4 (Fig. 3E, left panel). Further, siRNA mediated specific HIF1 $\alpha$ inhibition (Fig. 3E, middle panel) or treatment with HIF1 $\alpha$ inhibitor 2-methoxy estradiol reduced HE4 expression concomitantly (Fig. 3E, right panel). Full length of the gels corresponding to Fig. 3 (middle and right are included in the supplementary section Fig. 3.

\section{Discussion}

HE4 overexpression with serum levels $>150 \mathrm{pm}$ is common in $78 \%$ of ovarian cancer patients as compared to breast (13\%), endometrial (25\%), gastrointestinal (16\%) and lung (42\%) tumors ${ }^{21}$. High sensitivity and specificity of HE4 serum expression in EOC patients had led to the USFDA approval of HE4 as a biomarker for ovarian cancer. Similarly, the ROMA algorithm was approved to detect ovarian tumor among the women presenting with a pelvic mass or cyst ${ }^{9,10}$.

The biological functions of HE4 despite recent implications in the immune defense $\mathrm{e}^{22,23}$ remain elusive. Previous reports attribute a role 


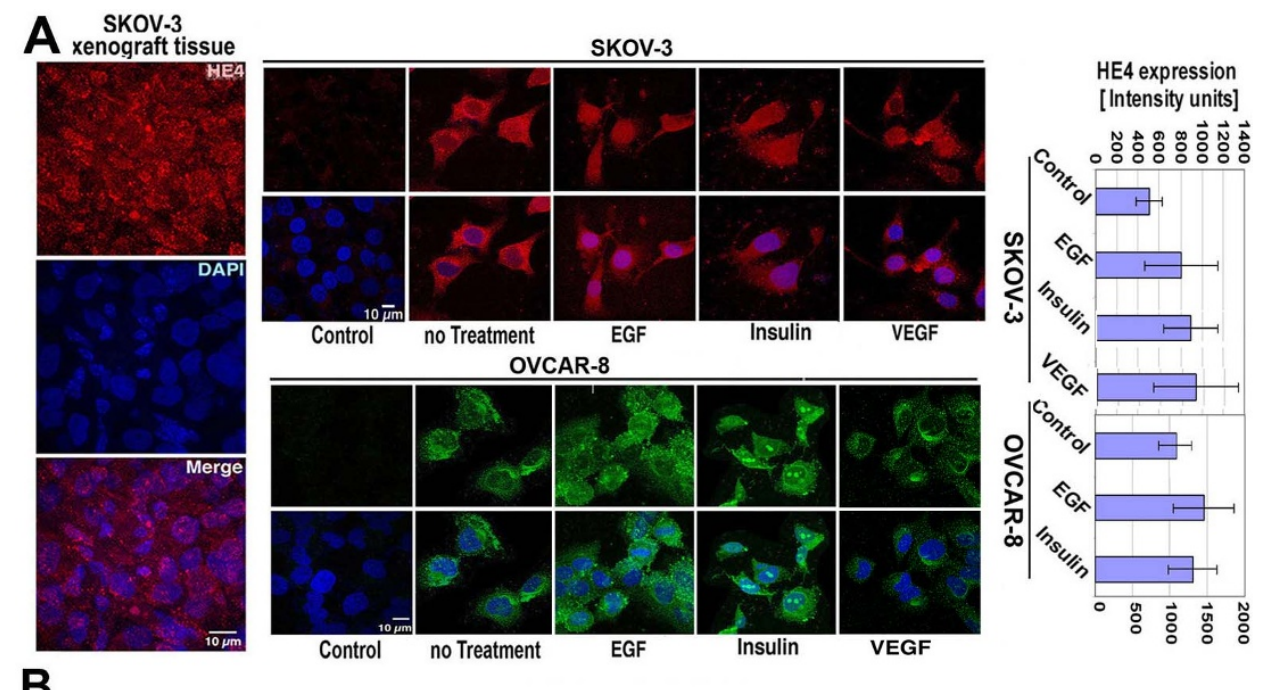

B
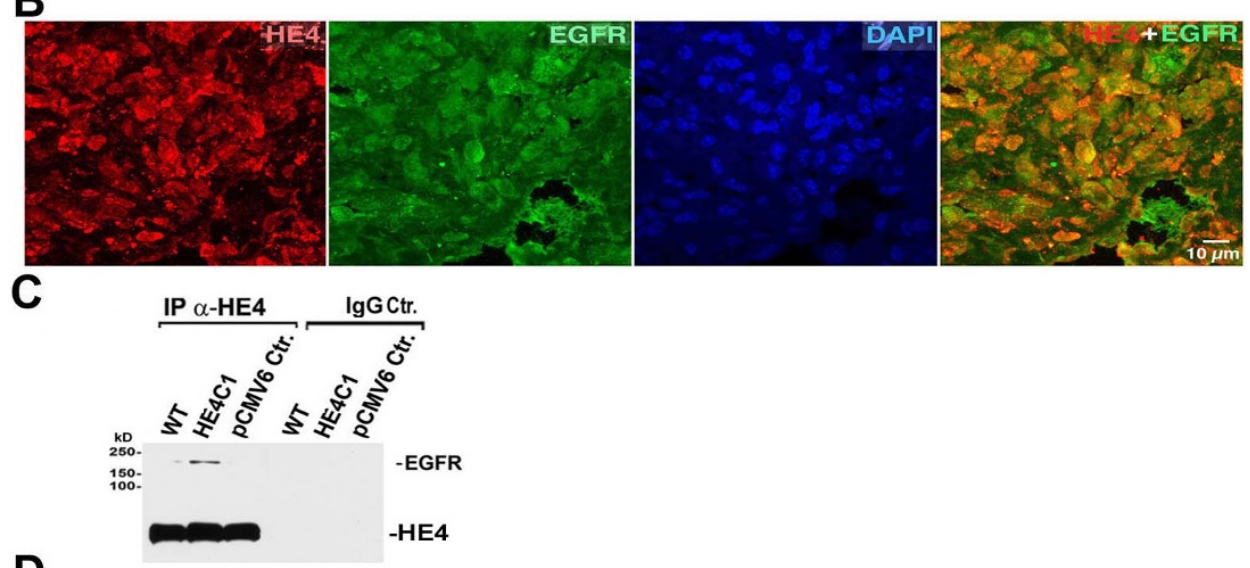

D

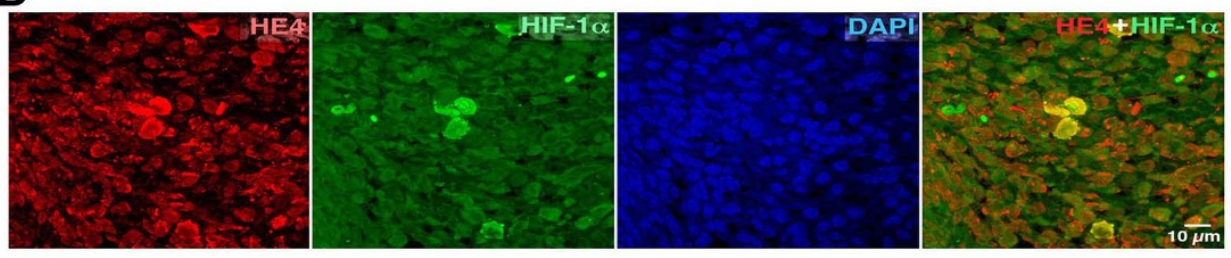

E
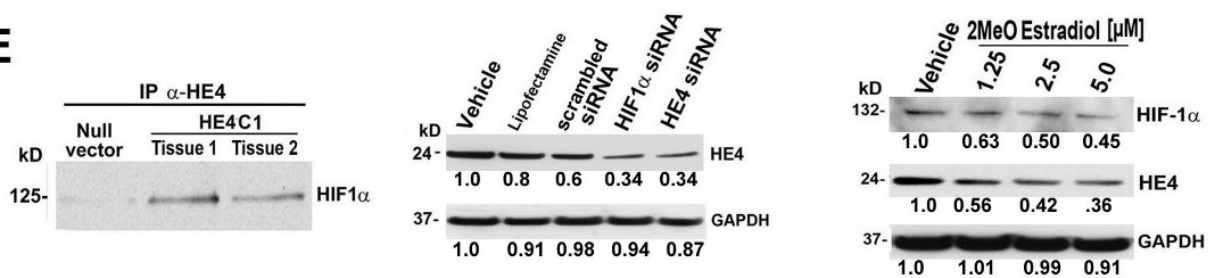

Figure 3 HE4 associates with EGFR and HIF1a. (A) Confocal images of SKOV-3 xenograft tissue stained with HE4 primary antibody and corresponding Alexa Fluor secondary antibody and chromatin staining (DAPI). Intense nuclear HE4 staining was observed (left panel); Bar=10 $\mu \mathrm{M}$. Confocal microscopy of serum starved SKOV-3 (upper middle panel) or OVCAR-8 (lower middle panel) cells upon stimulation with EGF (33 ng/ml), insulin $(83 \mathrm{ng} / \mathrm{ml})$ or VEGF $(16.6 \mathrm{ng} / \mathrm{ml})$ for 30 minutes showed intensified nuclear HE4 localization and increased overall expression of HE4 (right panel; average intensity of 6 fields is shown) as compared to non-treated cells. (B) Paraffin embedded SKOV-3 xenograft tumor tissues were processed and stained with antibodies against HE4 and EGFR and images analyzed by immunofluorescence microscopy. Yellow spots indicate co-localization of HE4 with EGFR. (C) HE4 was immunoprecipitated from the lysates of SKOV-3 WT cells and western blot analysis performed. EGFR co-immunoprecipitation was detected and increased in HE4 overexpressing HE4C1. (D) Paraffin embedded SKOV-3 derived xenograft tumor tissues were processed and stained with antibodies against HE4 and HIF1 $\alpha$ and immunofluorescence microscopy carried out. Yellow spots indicate co-localization of HE4 with HIF1 $\alpha$. (E) Lysates of HE4C1 and null vector transformed SKOV-3 derived xenograft tumors were immunoprecipitated with HE4 primary antibody and probed for HIF1 $\alpha$ by immunoblotting revealing HIF1 $\alpha$ coprecipitation along with increased HE4 overexpression (left panel). HIF1 $\alpha$ and HE4 siRNA treatment inhibited HE4 expression in SKOV-3 cells (middle panel). Lipofectamine and scrambled oligo were used as control. Treatment of SKOV-3 for 24 h with a HIF1 $\alpha$ inhibitor (2MeOEstradiol) resulted in decreased HE4 expression (right panel).Gels were run in similar conditions. Full length gels corresponding to middle and right panel are shown in Supplementary Information section (Fig. 3, Supplementary Info).Only single bands were detected. Densitometric analysis of the immunoblots was carried out and the values relative to control (set to 1) are shown. 
of HE4 expression in ovarian cancer cell line adhesion and motility ${ }^{24}$ as well as in endometrial tumor growth in an animal model ${ }^{14}$. HE4 has also been shown to be a sensitive biomarker in renal fibrosis and inhibition of HE4 expression via a neutralizing antibody resolved kidney fibrosis in an animal model ${ }^{25,26}$. Our study demonstrates the impact of HE4 overexpression on ovarian cancer proliferation and on chemotherapy both in vitro and in an animal model. HE4 overexpressing cells derived from SKOV-3 and OVCAR-8 cell lines exhibited a diminished response to cisplatin and paclitaxel. In nude mice, xenografts derived from an HE4 overexpressing SKOV-3 clone formed larger tumors than the control groups during the 20 days of trial and subsequent treatment with cisplatin revealed development of drug resistance. These studies are concordant with serum HE4 levels and survival outcomes of 89 EOC patients that were examined for this study. We observed that patients with platinum resistant disease or HE4 overexpression both displayed lower survival compared to the platinum sensitive or low HE4 expressing groups $(\mathrm{p}=0.001)$. A previously published OVCAD study ${ }^{27}$ and a study by Kang et al. ${ }^{28}$ suggests a correlation of HE4 levels with platinum resistance and poor survival in ovarian cancer patients.

Our data identify HE4 as a selective molecular target to suppress ovarian cancer cell viability in vitro and tumor growth in vivo via PTO or alternative methods. Confocal microscopy revealed nuclear staining of HE4 in SKOV-3 xenograft tissues. Similarly, a previous study by Georgakopoulos et al. ${ }^{29}$ suggested partial nuclear localization of HE4 in human ovarian tumor tissues. In contrast, cultured ovarian cancer cells demonstrated cytosolic staining. This observation led us to investigate plausible factors that may induce nuclear translocation of HE4. Our experiments reveal that the spatial expression of HE4 is linked to the activity of epidermal growth factor (EGF), vascular endothelial growth factor (VEGF) and insulin, which induce nuclear or in the case of insulin also nucleolar translocation. Recent studies have suggested a role of the nucleolus in cancer progression and revealed oncogenes other than HE4 localizing to nucleoli such as p53, RB protein, c-Myc which target ribosomal biosynthesis in nucleoli ${ }^{30-32}$. EGF, VEGF and Insulin and their receptors are directly linked to ovarian tumor growth and chemoresistance ${ }^{33-35}$. The effect of VEGF on HE4 nuclear translocation suggested a role for HE4 in the angiogenic component of the tumor microenvironment. VEGF is essential for hypoxia-inducible factormediated neovascularization and regulated by the hypoxia-inducible factor (HIF) family ${ }^{36}$. Outcomes of immunoprecipitation assays, colocalization and application of HIF1 $\alpha$ inhibitor 2-methoxyestradiol and siRNA revealed the interaction of HE4 with HIF1 $\alpha$. As for HIF $1 \alpha$, colocalization and coimmunoprecipitation of EGFR with HE4 supported interaction of HE4 with the cognate receptor of EGF.

Collectively, our data for the first time, implicate HE4 overexpression as a molecular driver for tumor growth and chemoresistance as reflected in decreased survival rates for ovarian cancer patients with tumors that overexpress HE4. We show evidence that HE4 expression and localization is correlated with the function of growth factors. Our study demonstrates that HE4 expression or interactions are potential targets for the treatment of ovarian cancer.

\section{Methods}

Cell culture. Human cell lines SKOV-3 (ovarian adenocarcinoma), OVCAR-3 (ovarian epithelial adenocarcinoma), and OVCAR-8 (ovarian adenocarcinoma) were obtained from American Type Culture Collection (Manassas, VA). Cells were grown T75 cell culture flasks (Corning, New York, NY) in complete DMEM medium (Gibco, Rockville, MD) supplemented with $10 \%$ fetal bovine serum and $1 \%$ pen/strep antibiotic according to the suppliers recommendation.

Cell viability assay. Viability of cells before and after drug treatment was determined was determined by the $96{ }^{\circledR}$ Aqueous-One-Solution Assay (Promega, Madison, WI). Briefly, cells (5000/well) were plated into 96 well flat bottom plates (Corning, Inc., Corning, NY) before treatment with solution of cisplatin or paclitaxel in DMSO or vehicle (DMSO) as indicated. Following incubation at $37^{\circ} \mathrm{C}$ in a cell culture incubator for $20 \mathrm{~h}$ MTS reagent was added at a 1:10 dilution to the medium. The samples were incubated for an additional $4 \mathrm{~h}$ before absorbance was measured at $490 \mathrm{~nm}$ in an
ELISA plate reader (Thermo Labsystems, Waltham, MA). Experiments were performed in triplicates; data are expressed as the mean of the triplicate determinations $(\mathrm{X} \pm \mathrm{SD})$ of a representative experiment in \% of absorbance by samples with untreated cells $(=100 \%)$.

Development of HE4 overexpressing clones for in vitro and in vivo studies. An HE4 overexpressing vector for stable expression was engineered by inserting the coding sequence of human WAP four-disulfide core domain 2 (WFDC2) cDNA into eukaryotic expression vector, pCMV6-entry (Origene, Rockville, MD). Transfection of the constructs in SKOV-3 and OVCAR- 8 cells was performed using Lipofectamine 2000 (Invitrogen, Carlsbad, CA). Stably transfected cells were selected by resistance to G-418 $(0.5 \mu \mathrm{g} / \mathrm{ml})$ (Research Products International, Mount Prospect, IL), applied $48 \mathrm{~h}$ after transfection and continued during cell culture. G-418 resistant cells were seeded as $200 \mu \mathrm{l} /$ well on a 96 well cell culture plate (Corning; New York, NY). Cells growing from single colonies (cloned; stable transfection) were isolated. Multiple clones were selected and cellular or secretory HE4 levels among clones were measured by ELISA (Fujirebio Inc; Philadelphia, PA) or PCR. SKOV-3, OVCAR-8 and the null vector or HE4 overexpressing clone xenograft experiments were performed as described previously ${ }^{37}$.

Stimulations and confocal microscopy experiments. SKOV- 3 and OVCAR- 8 cells were grown to semi-confluence in complete DMEM medium in a lab-Tek 8 chamber slides. Cells were serum starved overnight and were stimulated with VEGF (16.6 ng/ $\mathrm{ml})$, EGF (33 ng/ml) or insulin $(83 \mathrm{ng} / \mathrm{ml})$ for 30 minutes. Cells were fixed with $10 \%$ neutral buffered formalin and washed with PBST two times. Fixed cells were incubated with rabbit HE4 primary antibody (HE4 rabbit mAb, cat no-TA307787, Origene, Austin TX;1:1000) overnight at $4^{\circ} \mathrm{C}$, washed with PBST $(3 \times 5 \mathrm{~min})$ and incubated with Alexa Fluor 488 or 546 secondary antibody $(1: 2000)$ for 2 hours at room temperature in the dark. Slides were washed $(5 \times 5 \mathrm{~min})$ and DAPI containing mounting medium was applied. For co-localization studies paraffin embedded SKOV-3 derived xenograft tissues were processed and stained with primary HE4, EGFR (cat no-4405; Cell Signaling, Danvers, MA) or HIF1 $\alpha$ (cat no-13515; Santa Cruz biotechnology, Santa Cruz, CA) antibody for 24 hours. Cells were washed and Alexa Flour 488 and 546 antibody was applied. Confocal images were obtained and processed as published earlier ${ }^{37}$.

Coimmunoprecipitation experiments. SKOV-3, null vector and $\mathrm{HE} 4$

overexpressing clones HE4C1 and HE4C7 cells were seeded into $100 \mathrm{~mm}^{2}$ dishes and cultured to $\sim 80 \%$ confluency. Cells were rinsed in PBS, $\mathrm{pH} 7.4$, scraped off in $1 \mathrm{x}$ lysis buffer (20 mM Tris-HCl, pH 7.5) $150 \mathrm{mM} \mathrm{NaCl}, 1 \mathrm{mM}$ Sod-EDTA, 1 mM EGTA, $1 \%$ Triton, $2.5 \mathrm{mM}$ Sodium pyrophosphate, $1 \mathrm{mM}$ b-glycerophosphate, $1 \mathrm{mM}$ Sodium Vanadate, $1 \mu \mathrm{g} / \mathrm{ml}$ Leupeptin, $1 \mathrm{mM}$ PMSF. Lysates were rocked at $4{ }^{\circ} \mathrm{C}$ for $5 \mathrm{~min}$, sonicated (10 pulses, $5 \mathrm{sec}$ ), centrifuged at $14000 \mathrm{~g}$ for $10 \mathrm{~min}$ and protein concentration of the supernatant quantitated (BioRad Protein estimation kit, Hercules, CA). Lysates were adjusted to $1 \mu \mathrm{g} / \mu \mathrm{l}$ total protein concentration in the original lysis buffer and $200 \mu \mathrm{l}$ lysates per sample used for each IP reaction. Identical samples were prepared for pulldown antibody (HE4, cat no-TA307787, Origene); EGFR (cat no-4405) or IGF1 $\beta$ receptor (cat no-6113, Cell Signaling, Danvers, MA) and Isotype IgG control (cat no- $3900 \mathrm{~s}$, Cell Signaling) with Ab concentration identical at $40 \mathrm{ng} / \mu \mathrm{l} .40 \mu \mathrm{l}$ per sample of Protein G Sepharose (Invitrogen, Carlsbad, CA) $50 \%$ bead slurry were washed 3 times in $200 \mu \mathrm{l}$ PBS and microcentrifuged at $10.000 \mathrm{rpm}$ for $1 \mathrm{~min}$ ). Beads were resuspended with Ab in PBS buffer, incubated at $4{ }^{\circ} \mathrm{C}$ overnight and washed $3 \mathrm{x}$ in $400 \mu \mathrm{l}$ PBS. Beads were added to each $200 \mu$ l lysate sample, incubated at $4{ }^{\circ} \mathrm{C}$ on a rotator for $4 \mathrm{~h}$, spun and washed $3 \mathrm{x}$ in $400 \mu \mathrm{l}$ lysis buffer with $150 \mathrm{mM}$ and $2 \mathrm{x}$ with $300 \mathrm{mM} \mathrm{NaCl}$. The beads were resuspended in $20 \mu \mathrm{l}$ Laemli buffer, vortexed, heated to $95^{\circ} \mathrm{C}$ for $3 \mathrm{~min}$, spun and the supernatant loaded for PAGE analysis.

1. Kirchhoff, C., Habben, I., Ivell, R. \& Krull, N. A major human epididymis-specific cDNA encodes a protein with sequence homology to extracellular proteinase inhibitors. Biol Reprod 45, 350-357 (1991).

2. Bingle, L., Singleton, V. \& Bingle, C. D. The putative ovarian tumour marker gene HE4 (WFDC2), is expressed in normal tissues and undergoes complex alternative splicing to yield multiple protein isoforms. Oncogene 21, 2768-2773 (2002).

3. Drapkin, R. et al. Human epididymis protein 4 (HE4) is a secreted glycoprotein that is overexpressed by serous and endometrioid ovarian carcinomas. Cancer Res 65, 2162-2169 (2005)

4. Galgano, M. T., Hampton, G. M. \& Frierson, H. F. Jr. Comprehensive analysis of HE4 expression in normal and malignant human tissues. Mod Pathol 19, 847-853 (2006).

5. Hellström, I. et al. The HE4 (WFDC2) protein is a biomarker for ovarian carcinoma. Cancer Res 63, 3695-6700 (2003).

6. Ono, K. et al. Identification by cDNA microarray of genes involved in ovarian carcinogenesis. Cancer Res 60, 5007-5011 (2000).

7. Welsh, J. B. et al. Analysis of gene expression profiles in normal and neoplastic ovarian tissue samples identifies candidate molecular markers of epithelial ovarian cancer. Proc Natl Acad Sci 98, 1176-1181 (2001).

8. Huhtinen, K. et al. Serum HE4 concentration differentiates malignant ovarian tumors from ovarian endometriotic cysts. Br J Cancer 100, 1315-1319 (2009). 
9. Moore, R. G. et al. Comparison of a novel multiple marker assay vs the Risk of Malignancy Index for the prediction of epithelial ovarian cancer in patients with a pelvic mass. Am J Obstet Gynecol 203, 228. e1-6 (2010).

10. Moore, R. G. et al. A novel multiple marker bioassay utilizing HE4 and CA 125 for the prediction of ovarian cancer in patients with a pelvic mass. Gyn Oncol 112, 40-46 (2009).

11. Van Gorp, T. et al. HE4 and CA 125 as a diagnostic test in ovarian cancer: prospective validation of the Risk of Ovarian Malignancy Algorithm. Br J Cancer 104, 863-870 (2011).

12. Montagnana, M. et al. The ROMA (Risk of Ovarian Malignancy Algorithm) for estimating the risk of epithelial ovarian cancer in women presenting with pelvic mass: is it really useful? Clin Chem Lab Med 49, 521-525 (2011).

13. Molina, R. et al. HE4 a novel tumour marker for ovarian cancer: comparison with CA 125 and ROMA algorithm in patients with gynaecological diseases. Tumour Biol 32, 1087-1095 (2011).

14. Li, J. et al. HE4 (WFDC2) Promotes tumor growth in endometrial cancer cell lines. Int J Mol Sci 14, 6026-6043 (2013).

15. Oliver, T. G. et al. Chronic cisplatin treatment promotes enhanced damage repair and tumor progression in a mouse model of lung cancer. Genes Dev 24, 837-852 (2010).

16. Sayer, R. A. et al. High insulin-like growth factor-2 (IGF-2) gene expression is an independent predictor of poor survival for patients with advanced stage serous epithelial ovarian cancer. Gyn Oncol 96, 355-361 (2005).

17. Beck, E. P. Identification of insulin and insulin-like growth factor I (IGF I) receptors in ovarian cancer tissue. Gyn Oncol 53, 196-201 (1994).

18. Osada, R et al. Expression of hypoxia-inducible factor 1alpha, hypoxia-inducible factor 2alpha, and von Hippel-Lindau protein in epithelial ovarian neoplasms and allelic loss of von Hippel-Lindau gene: nuclear expression of hypoxia-inducible factor lalpha is an independent prognostic factor in ovarian carcinoma. Hum Pathol 38, 1310-1320 (2007).

19. Nakai, H., Watanabe, Y., Ueda, H. \& Hoshiai, H. Hypoxia inducible factor 1-alpha expression as a factor predictive of efficacy of taxane/platinum chemotherapy in advanced primary epithelial ovarian cancer. Cancer Lett 251, 164-167 (2007).

20. Birner, P. M., Schindle, A., Obermair, G., Breitenecker, G. \& Oberhuber, G. Expression of hypoxia-inducible factor 1 alpha in epithelial ovarian tumors: its impact on prognosis and on response to chemotherapy. Clin Cancer Res 7, 1661-1668 (2001)

21. Moore, R. G., Brown, A. K., Miller, M. C. et al. The use of multiple novel tumor biomarkers for the detection of ovarian carcinoma in patients with a pelvic mass. Gynecol Oncol 108, 402-408 (2008).

22. Bingle, L. et al. WFDC2 (HE4): A potential role in the innate immunity of the oral cavity and respiratory tract and the development of adenocarcinomas of the lung. Respiratory Research 7, 61 (2006)

23. Wei, H. et al. Silencing of the TGF- $\beta 1$ Gene Increases the Immunogenicity of Cells from Human Ovarian Carcinoma. J Immunother 35, 267-275 (2012).

24. Lu, R. et al. Human epididymis protein 4 (HE4) plays a key role in ovarian cancer cell adhesion and motility. Biochem Biophys Res Commun 419, 274-280 (2012).

25. Allison, S. J. Fibrosis: HE4-a biomarker and target in renal fibrosis. Nature Reviews Nephrology 9, 124 (2013).

26. LeBleu, V. S. et al. Identification of human epididymis protein-4 as a fibroblastderived mediator of fibrosis. Nature Medicine 19, 227-231 (2013).

27. Braicu, E. I. et al. Preoperative HE4 overexpresssion in plasma predicts surgical outcome in primary ovarian cancer patients. Results from the OVCAD study. Gynecol Oncol 128, 245-251 (2013).
28. Kang, S. et al. Serum HE4 level is an independent risk factor of surgical outcome and prognosis of epithelial ovarian cancer. Gyn Oncol 120, S2-S133 (2011).

29. Georgakopoulos, P., Mehmood, S., Akalin, A. \& Shroyer, K. R. Immunohistochemical localization of HE4 in benign, borderline and malignant lesion of the ovary. Int J of Gyn Path 31, 517-523 (2012).

30. Zhai, W. \& Comai, L. Repression of RNA polymerase I transcription by the tumor suppressor p53. Mol Cell Biol 20, 5930-5938 (2000).

31. Cavanaugh, A. H. et al. Activity of RNA polymerase I transcription factor UBF blocked by Rb gene product. Nature 374, 177-180 (1995).

32. Arabi, A. et al. c-Myc associates with ribosomal DNA and activates RNA polymerase I transcription. Nat Cell Biol 7, 303-310 (2005).

33. Maihle, N. J. et al. EGF/ErbB receptor family in ovarian cancer. Cancer Treat and Res 107, 247-258 (2002).

34. Gotlieb, W. H. et al. Insulin-like growthfactor receptor I targeting in epithelial ovarian cancer. Gynecol Oncol 100, 389-396 (2006).

35. Spannuth, W. A. et al. Functional significance of VEGFR-2 on ovarian cancer cells. Int J Cancer 124, 1045-1053 (2009).

36. Oladipupo, S. et al. VEGF is essential for hypoxia-inducible factor-mediated neovascularization but dispensable for endothelial sprouting. Proc Natl Acad Sci 108, 13264-13269 (2011).

37. Moore, R. G. et al. Efficacy of a Non-Hypercalcemic Vitamin-D2 Derived AntiCancer Agent (MT19c) and Inhibition of Fatty Acid Synthesis in an Ovarian Cancer Xenograft Model. PLoS ONE 7, e34443 (2012).

\section{Acknowledgments}

This study was supported by a 'Swim Across America' grant to R.G.M. and R.K.S. R.G.M. is also supported by NCI grant RO1 CA136491-01. The authors thank Ginny Hovanesian for help with microscopy.

\section{Author Contributions}

R.K.S. and R.G.M. conceived and designed the studies. Patient studies were conducted by R.G.M. and S.M. Statistical analysis was performed by M.C.M. Clones were developed by N.Y., Y.D.T. and J.F.P. Animal experiments, in vitro experiments and cell stimulations were performed by E.K.H., T.H. and R.K.S. ELISA analyses were performed by G.L.M. Immunoprecipitations were performed by T.S.L., K.K.K. and N.Y. Manuscript was written by R.K.S., T.S.L. and R.G.M. Every author read and approved the manuscript.

\section{Additional information}

Supplementary information accompanies this paper at http://www.nature.com/ scientificreports

Competing financial interests: R.G.M. and R.K.S. are listed as co-inventors on a pending patent application (US61/493881). Patent was assigned to Women and Infants Hospital of Rhode Island. Other authors declare that no competing interest exists.

How to cite this article: Moore, R.G. et al. HE4 (WFDC2) gene overexpression promotes ovarian tumor growth. Sci. Rep. 4, 3574; DOI:10.1038/srep03574 (2014).

This work is licensed under a Creative Commons AttributionNonCommercial-NoDerivs 3.0 Unported license. To view a copy of this license, visit http://creativecommons.org/licenses/by-nc-nd/3.0 